\title{
CURVES ON $K$-THEORY AND THE DE RHAM HOMOLOGY OF ASSOCIATIVE ALGEBRAS
}

\author{
JOHN G. RYAN
}

\begin{abstract}
This paper describes the generalization to arbitrary associative algebras of the complex of "typical curves on algebraic $K$-theory" and shows, in particular, that for certain Q-algebras, $A$, the complex is isomorphic to the "generalized de Rham complex," $\left(H H_{*}(A), B\right)$, in which $B$ is Connes' operator acting on the Hochschild homology groups of $A$.
\end{abstract}

\section{INTRODUCTION}

In [Bl], Bloch defined a cochain complex of "typical curves" on the algebraic $K$-theory of commutative $\mathbf{F}_{p}$-algebras. The background to Bloch's paper is the result of Van der Kallen that, for commutative $\mathbf{Z}[1 / 2]$-algebras $R$, the group

$$
\mathscr{C}_{2} K_{2}(R) \stackrel{\text { def }}{=} \operatorname{ker}\left(K_{2}\left(R[T] /\left(T^{2}\right)\right) \stackrel{T \mapsto 0}{\rightarrow} K_{2}(R)\right)
$$

is isomorphic to the group of Kaehler differentials $\Omega_{R / \mathbf{Z}}^{1}$, and the fact that the group

$$
\mathscr{C}_{\infty} K_{1}(R) \stackrel{\text { def }}{=} \lim _{\leftarrow n} \operatorname{ker}\left(K_{1}\left(R[T] /\left(T^{n}\right)\right) \stackrel{T_{\hookrightarrow}}{\rightarrow} K_{1}(R)\right)
$$

is isomorphic to the additive group $\mathscr{W}(R)$ of (Cartier's) generalized Witt vectors with coefficients in $R$. Cartier had shown that, for smooth connected commutative formal groups $G$ over a ring $R$, the role that is played by the Lie algebra of $G$ (which is just $\mathscr{C}_{2} G(R)$ in the above notation) when $R$ is a Q-algebra is played in general by the group (with operators) of "curves on $G$," $\mathscr{C}_{\infty} G(R)$. Bloch showed that, if $R$ is a commutative smooth local ring, then the "curves on the symbol part of $K$-theory" (i.e., the groups

$$
\mathscr{C}_{\infty} K_{p}^{\text {sym }}(R) \stackrel{\text { def }}{=} \lim _{\leftarrow n} \operatorname{ker}\left(K_{p}^{\text {sym }}\left(R[T] /\left(T^{n}\right)\right) \stackrel{T_{\hookrightarrow}}{\rightarrow} K_{p}^{\text {sym }}(R)\right)
$$

where the superscript "sym" denotes the subgroup generated by the generalized Steinberg symbols) are modules over $\mathscr{W}(R)$, and that there is a derivation

$$
\partial: \mathscr{C}_{\infty} K_{p}^{\mathrm{sym}}(R) \rightarrow \mathscr{C}_{\infty} K_{p+1}^{\mathrm{sym}}(R) .
$$

Received by the editors October 27, 1988.

1980 Mathematics Subject Classification (1985 Revision). Primary 18G35, 18F25; Secondary $19 \mathrm{D} 55$.

This work was partially supported by a Henry Rutgers Research Fellowship. 
As in the case of Cartier's curves on formal groups, the invertibility of a set of rational integers in $R$ allows one to define idempotents in the ring of operators on the curves and determines a direct product decomposition of the complex $\left(\mathscr{C}_{\infty} K_{p}^{\text {sym }}(R) ; \partial\right)$ into isomorphic "typical" pieces. Bloch showed that, if $R$ is a perfect field of characteristic $p>2$ and $X$ a smooth scheme over $R$, then the hypercohomology of $X$ with coefficients in the (sheafification of the) complex of $p$-typical curves on $K_{*}^{\text {sym }}\left(\mathscr{O}_{X}\right)$ is isomorphic to (Grothendieck's) "crystalline cohomology" of $X$. Recall that crystalline cohomology was devised as a generalization (for arbitrary bases and for not-necessarily smooth schemes) of the de Rham cohomology of smooth schemes over fields of characteristic zero (cf. [Gro]). Bloch's was the first work to show that, for smooth schemes (over $\mathbf{F}_{p}$ ), crystalline cohomology could be calculated as the hypercohomology of a cochain complex (rather than in terms of toposes or of local liftings to characteristic zero). Bloch's complex has been reconstructed by Illusie "without the $K$-theory" following Deligne's modification of earlier work by Lubkin (cf. [IIl]).

In another direction, Grayson and Stienstra (following work by Almqvist) have constructed complexes from the $K$-theory of the "exact category of endomorphisms over a commutative ring $R$," i.e., from the category whose objects are pairs $(M, \alpha)$, in which $M$ is a finitely generated projective $R$-module and $\alpha$ an endomorphism of $M$. The map that takes an endomorphism to its characteristic polynomial induces a map from the Grayson-Stienstra complex $\left(\widetilde{K}_{*}\left(\operatorname{End}_{R}\right), \partial\right)$ to Bloch's complex of curves $\left(\mathscr{C}_{\infty} K_{p}^{\text {sym }}(R) ; \partial\right)$. This leads to many simplifications of Bloch's arguments (cf. [St 1])

The organization of this paper is as follows. In $\S 1$, the complex of curves on $K$-theory is defined for arbitrary rings. The definitions are fairly straightforward modifications of those of Bloch. The proofs, however, rely on the pairing

$$
\mathscr{C}_{\infty} K_{p}(R) \times \widetilde{K}_{q}(\mathrm{Nil}(B)) \rightarrow K_{p+q}(R \otimes B),
$$

which is nondegenerate as $B$ varies over commutative rings. This brings to bear Steinstra's results, allowing easy proofs of the general properties of the complex.

$\S 2$ examines the case in which the ring $R$ is a $\mathbf{Q}$-algebra. Here, the crucial result needed is Goodwillie's theorem [Go 1] linking rational $K$-theory relative to a nilpotent ideal to rational relative cyclic homology. The conclusion of $\S 2$ is Theorem 2.8, which gives a simple homological interpretation of the complex of typical curves on the $K$-theory of $\mathbf{Q}$-algebras that satisfy a certain condition which I call the " $K$-theoretic $)$ curves condition."

Note. Chuck Weibel has pointed out to me that he had noted (in an unpublished letter to Stienstra) the fact that Goodwillie's theorem allows the determination of the groups $\mathscr{C}_{\infty} K_{*}(A)$, for $\mathbf{Q}$-algebras $A$. Thus, the results at the beginning of $\S 2.2$ are already well known to the experts. I would like to thank him for introducing me to the work of Stienstra and Bloch and for answering the many 
questions that I asked. I would also like to thank Gerhard Hochschild for his constant encouragement and advice.

\section{THE COMPLEX OF TYPICAL CURVES ON $K$-THEORY}

\subsection{The $V$ and $F$ operators.}

Definition (cf. [Bl]). Let $R$ be a ring, $m$ a positive integer. We define the group of curves of length $m$ on the algebraic $K$-theory of $R, \mathscr{C}_{m} K_{*}(R)$, by

$$
\mathscr{C}_{m} K_{*}(R)=\operatorname{ker}\left(K_{*}\left(R[T] /\left(T^{m}\right)\right) \stackrel{T^{\prime} 0}{\rightarrow} K_{*}(R)\right) .
$$

The groups $\mathscr{C}_{m} K_{*}(R)$ form a cofiltered diagram (= projective system) with respect to the maps induced by the natural ring homomorphisms:

$$
R[T] /\left(T^{M}\right) \rightarrow R[T] /\left(T^{m}\right) \quad(M \geq n) .
$$

We denote the limit of this diagram by $\mathscr{C}_{\infty} K_{*}(R)$, which we regard as a topological group (with the "projective limit topology"). We will refer to elements of $\mathscr{C}_{\infty} K_{*}(R)$ as, simply, curves on $K_{*}(R)$.

For each positive integer $n$, the ring homomorphisms

$$
\begin{aligned}
p_{n}: R[T] /\left(T^{m}\right) & \rightarrow R[T]\left(T^{n m}\right), \\
T & \mapsto T^{n}
\end{aligned}
$$

induce continuous endomorphisms $V_{n}$ and $F_{n}$ of $\mathscr{C}_{\infty} K_{*}(R): V_{n}$ is the limit of the maps

$$
\mathscr{C}_{m} K_{*}(R) \rightarrow \mathscr{C}_{n m} K_{*}(R)
$$

induced (directly) by $p_{n} ; F_{n}$ is the limit of the maps

$$
\mathscr{C}_{n m} K_{*}(R) \rightarrow \mathscr{C}_{m} K_{*}(R)
$$

which are the transfer maps associated with $p_{n}$.

Proposition 1.1. The following identities hold for the operations $F_{n}$ and $V_{n}$ on the group of curves on $K_{*}(R)$, for an ring $R$ :

(1) $V_{1}=F_{1}=1 ; F_{n} \circ V_{n}=n(=$ multiplication by $n) ; F_{m} \circ F_{n}=F_{n m}$; $V_{n} \circ V_{m}=V_{n m}$ (for all positive integers $\left.n, m\right)$.

(2) If $m$ and $n$ are relatively prime then

$$
V_{n} \circ F_{m}=F_{m} \circ V_{n} \text {. }
$$

(3) If $R$ is an $\mathbf{F}_{p}$-algebra, then $V_{p} \circ F_{p}=p$.

Proof. The relations in (1) are obvious from the definitions; those of (2) and (3) will be proved in Corollary 2.3 below.

To prove assertions (2) and (3) of Proposition 2.1 (as well as other relations between operators on curves) we need to define (as in [BI]) a pairing between curves on the $K$-theory of a ring $R$ and the $K$-groups of a category of nilpotent endomorphisms. 
Definition. Let $B$ be a ring. Let $\operatorname{Nil}(B)$ denote the exact category whose objects are pairs $(M, \beta)$, in which $M$ is a finitely generated projective $B$-module and $\beta$ a nilpotent endomorphism of $M$. Morphisms $\phi:(M, \beta) \rightarrow(N, \alpha)$ in $\operatorname{Nil}(B)$ are $B$-linear maps $\phi: M \rightarrow N$ satisfying $\alpha \circ \phi=\phi \circ \beta$; exact sequences in $\operatorname{Nil}(B)$ are determined by the exactness of the underlying sequence of morphisms of $B$-modules.

$\operatorname{Nil}(B)$ is filtered by the exact full subcategories $\operatorname{Fil}_{n}(\operatorname{Nil}(B))$, whose objects are those pairs $(M, \beta)$ for which $\beta^{n}=0$. The $K$-groups of $\operatorname{Nil}(B)$ are, thus, (as in [Q]) filtered colimits (= "inductive limits") of the $K$-groups of the $\operatorname{Fil}_{n}(\operatorname{Nil}(B))$ 's.

Now, for any rings $R$ and $B$ and any positive integer $n$, there is a biexact functor

defined by

$$
\mathscr{P}\left(R[T] /\left(T^{n}\right)\right) \times \operatorname{Fil}_{n} \operatorname{Nil}(B) \rightarrow \mathscr{P}\left(R \otimes_{\mathbf{Z}} B\right)
$$

$$
(M,(N, \beta)) \mapsto M \otimes_{\mathrm{Z}[T] / T^{n}} N_{\beta}
$$

where $N_{\beta}$ denotes $N$ viewed as a $\mathrm{Z}[T] /\left(T^{n}\right)$-module via $\beta$ and where, as usual, $\mathscr{P}(A)$ denotes the exact category of finitely generated projective $A$ modules for any ring $A$.

Via Waldhausen's $Q Q$-construction (see, for example, [Gr 1]) there is an induced linear map:

$$
\theta_{n}: K_{p}\left(R[T] / T^{n}\right) \otimes_{\mathbf{Z}} K_{q}\left(\operatorname{Fil}_{n} \operatorname{Nil}(B)\right) \rightarrow K_{p+q}\left(R \otimes_{\mathbf{Z}} B\right) .
$$

The $\theta_{n}$ 's are coherent with respect to the maps induced by the natural ring homomorphisms:

$$
R[T] /\left(T^{M}\right) \rightarrow R[T] /\left(T^{m}\right) \quad(M \geq m)
$$

and the inclusions

$$
\operatorname{Fil}_{m}(\operatorname{Nil}(B)) \hookrightarrow \operatorname{Fil}_{M}(\operatorname{Nil}(B)) .
$$

Thus, there is a linear map (functorial in $R$ and $B$ )

$$
\theta: \lim _{\leftarrow n} K_{p}\left(R[T] / T^{n}\right) \otimes_{\mathbf{Z}} K_{q}(\operatorname{Nil}(B)) \rightarrow K_{p+q}\left(R \otimes_{\mathbf{Z}} B\right) .
$$

There is an obvious split inclusion:

$$
\mathscr{P}(B) \leftrightarrows \mathrm{Nil}(B)
$$

If we denote by $\widetilde{K}_{*}(\operatorname{Nil}(B))$ the kernel of $K_{*}(\operatorname{Nil}(B)) \rightarrow K_{*}(B)$, then the pairing $\theta$ gives rise to a natural pairing, which we also denote by $\theta$ :

$$
\theta: \mathscr{C}_{\infty} K_{p}(R) \otimes_{\mathbf{Z}} \widetilde{K}_{q}(\operatorname{Nil}(B)) \rightarrow K_{p+q}\left(R \otimes_{\mathbf{Z}} B\right) .
$$

The crucial point to note about the above pairing $\theta$ is that it is nondegenerate, even as $B$ varies over only commutative rings. Specifically, if $\alpha \in \mathscr{C}_{\infty} K_{p}(R)$ is such that the image $\pi_{n} \alpha$ of $\alpha$ in $\mathscr{C}_{n} K_{p}(R)$ is nonzero, then, if $\beta_{n}$ is the element $\left[\left(\mathbf{Z}[T] / T^{n}, T\right)\right]-\left[\left(\mathbf{Z}[T] / T^{n}, 0\right)\right]$ of $\widetilde{K}_{0}\left(\operatorname{Nil}\left(\mathbf{Z}[T] / T^{n}\right)\right)$, we see that $\theta\left(\alpha \otimes \beta_{n}\right)=\pi_{n} \alpha$. 
A consequence of this is that, if we define a filtration on $\mathscr{C} K_{*}(R)$ by

Fil $_{n} \mathscr{C}_{\infty} K_{p}(R)=\left\{\alpha \in \mathscr{C}_{\infty} K_{p}(R): \forall\right.$ commutative rings $B$,

$$
\left.\forall \beta \in \widetilde{K}_{q}\left(\operatorname{Fil}_{n} \operatorname{Nil}(B)\right), \theta_{n}\left(\pi_{n} \alpha \otimes \beta\right)=0\right\},
$$

then

$$
\operatorname{Fil}_{n} \mathscr{C}_{\infty} K_{p}(R)=\operatorname{ker}\left(\mathscr{C}_{\infty} K_{*}(R) \stackrel{\pi_{n}}{\rightarrow} \mathscr{C}_{n} K_{*}(R)\right) .
$$

In other words, the pairing with $\widetilde{K}_{*}($ Nil $B)$ for commutative rings $B$ determines the topology on $\mathscr{C}_{\infty} K_{*}(R)$.

We can define operators $V_{n}$ and $F_{n}$ on $K_{*}(\operatorname{Nil}(B))$ for any ring $B$ as follows (cf. [Gr 2]). $F_{n}$ is induced by the exact functor

$$
\begin{aligned}
\operatorname{Fil}_{n m} \operatorname{Nil}(B) & \rightarrow \operatorname{Fil}_{m} \operatorname{Nil}(B), \\
(M, \beta) & \mapsto\left(M, \beta^{n}\right) .
\end{aligned}
$$

$V_{n}$ is induced by the exact functor

$$
\begin{array}{r}
\operatorname{Fil}_{m} \operatorname{Nil}(B) \rightarrow \operatorname{Fil}_{n m} \operatorname{Nil}(B), \\
(M, \beta) \mapsto\left(M^{\oplus n},\left[\begin{array}{cccc}
0 & 0 & \cdots & \beta \\
1 & 0 & \cdots & 0 \\
0 & \ddots & 0 & 0 \\
0 & \cdots & 1 & 0
\end{array}\right]\right) .
\end{array}
$$

Note that $V_{n}$ and $F_{n}$ stabilize the image of $K_{*}(B)$ : this is obvious for $F_{n}$; for $V_{n}$, we note that

$$
\left(M^{\oplus n},\left[\begin{array}{cccc}
0 & 0 & \cdots & 0 \\
1 & 0 & \cdots & 0 \\
0 & \ddots & 0 & 0 \\
0 & \cdots & 1 & 0
\end{array}\right]\right)
$$

has a filtration with quotients $(M, 0)$-the stability of $K_{*}(B)$ now follows from Quillen's resolution theorem [Q].

Lemma 1.2. Let $R$ and $B$ be rings, $\alpha \in \mathscr{C}_{\infty} K_{p}(R), \beta \in \widetilde{K}_{q}(\operatorname{Nil}(B)), n \geq 1$; the the pairing $\theta$ satisfies

$$
\theta\left(V_{n} \alpha \otimes \beta\right)=\theta\left(\alpha \otimes F_{n} \beta\right)
$$

and

$$
\theta\left(F_{n} \alpha \otimes \beta\right)=\theta\left(\alpha \otimes V_{n} \beta\right) .
$$

The proof is immediate from the definitions.

Corollary 1.3. The above lemma and the nondegeneracy of the pairing $\theta$ allow us to use Stienstra's results (Theorem 1.8 of [St 1]) on the operators $V_{n}$ and $F_{n}$ on $\widetilde{K}_{*}(\mathrm{Nil}(B))$ (for commutative rings $B$ ) to prove the remaining relations of Proposition 2.1.

1.2. Typification. If $R$ is a commutative ring and $A$ a central $R$-algebra (i.e., if the image of $R$ is contained in the center of $A$ ), then $\mathscr{C}_{\infty} K_{*}(A)$ is a (topological) module over the Cartier ring $\operatorname{Cart}(R)$ (cf. [Laz]). Recall that $\operatorname{Cart}(R)$ 
is the topological ring whose elements are infinite sums of the form

$$
\sum_{m, n \geq 1} V_{m}\left[r_{m, n}\right] F_{n}
$$

in which $r_{m, n} \in R$ and, $\forall n, r_{m, n}=0$ if $n \gg 0$. The action of the elements $[r]$ on curves is via "homotheties," i.e., via the homomorphisms

$$
\begin{aligned}
A[T] / T^{n} & \rightarrow A[T] / T^{n}, \\
T & \mapsto r T .
\end{aligned}
$$

Note that the operation of an element of the form $(*)$ on a curve in $\mathscr{C}_{\infty} K_{*}(A)$ is defined (this follows from the fact that the topology on $\mathscr{C}_{\infty} K_{*}(A)$ is determined by the pairing with the $K$-groups of $\operatorname{Nil}(B)$ for commutative algebras $B$, from Lemma 2.2, and from the fact that $\forall \beta \in \widetilde{K}_{q}\left(\operatorname{Nil}(B), F_{n} \beta=0, \forall n \gg 0\right)$.

One of the crucial points about the Cartier ring $\operatorname{Cart}(R)$ is that invertible (rational) integers in $R$ determine idempotents in $\operatorname{Cart}(R)$; specifically, if $\mathscr{U}$ is a set of rational primes that are invertible in $R$, and if $\langle\mathscr{U}\rangle$ is the multiplicative semigroups generated by $\mathscr{U}$, then the element

$$
\pi_{\langle\mathscr{U}\rangle} \stackrel{\text { def }}{=} \sum_{n \in\langle\mathscr{U}\rangle} V_{n}[\mu(n) / n] F_{n}
$$

(in which $\mu$ is the Möbius function) is an idempotent in $\operatorname{Cart}(R)$, and the image of the map "left multiplication by $\pi_{\langle\mathscr{U}\rangle}$ " coincides with $\bigcap_{n \in\langle\mathscr{U}\rangle} \operatorname{ker}\left(\right.$ left mult. by $\left.F_{n}\right)$.

Definition. If $S$ is the complement of a set $\mathscr{U}$ of rational primes that are invertible in a ring $R$ and if $A$ is a central $R$-algebra, then the group of $S$ typical curves on the $K$-theory of $A$, denoted $\mathscr{T}^{s} \mathscr{C}_{\infty} K_{*}(A)$, is the image of the (projection) map $\pi_{\langle\mathscr{U}\rangle}$ on $\mathscr{C}_{\infty} K_{*}(A)$.

Lemma 1.4 (Cartier). In the above notation, if $p \in S$ then

$$
F_{p} \circ \pi_{\langle\mathscr{U}\rangle}=\pi_{\langle\mathscr{U}\rangle} \circ F_{p}
$$

and

$$
V_{p} \circ \pi_{\langle\mathscr{U}\rangle}=\pi_{\langle\mathscr{U}\rangle} \circ V_{p} .
$$

1.3. The derivation. To define the derivation $\partial$ that makes the family of groups $\mathscr{C}_{\infty} K_{*}(R)$ into a cochain complex, we need to recall the role of the groups $K_{*}(\mathrm{Nil}(B))$ in the localization theorem of $K$-theory. According to Quillen's proof of the localization theorem [GQ], if $B$ is any ring and if $\mathscr{P}_{1}(B)$ denotes the exact category of finitely presented $B$-modules of projective dimension $\leq 1$, then

$$
Q \operatorname{Nil}(B) \stackrel{v}{\rightarrow} Q \mathscr{P}_{1}(B[X]) \rightarrow Q \mathscr{P}_{1}\left(B\left[X, X^{-1}\right]\right)
$$

is a homotopy-fibration. ( $Q$ is Quillen's $Q$-construction [Q]; the arrow $v$ is induced by the exact functor

$$
\begin{aligned}
\operatorname{Nil}(B) & \rightarrow \mathscr{P}_{1}(B[X]), \\
(M, \beta) & \mapsto M_{\beta} .
\end{aligned}
$$


Topological terminology about categories and functors should be interpreted as referring to the induced maps on classifying spaces.)

Let $\rho_{*}$ denote the connecting homomorphism

$$
\rho_{*}: K_{*}\left(B\left[X, X^{-1}\right]\right) \rightarrow K_{*-1}(\mathrm{Nil}(B))
$$

in the homotopy sequence of the homotopy-fibration $(\dagger)$. Now, if we specialize to the base $B=\mathrm{Z}[T] / T^{n}$, we can define a derivation

$$
\partial^{(n)}: \mathscr{C}_{\infty} K_{p}(R) \rightarrow \mathscr{C}_{n} K_{p+1}(R)
$$

as follows:

$$
\partial^{(n)}(\alpha)=\theta\left(\alpha \otimes \rho_{2}\left\{X^{-1}, 1-X^{-1} T\right\}\right),
$$

where $\rho_{2}\left\{X^{-1}, 1-X^{-1} T\right\} \in \widetilde{K}_{1}\left(\mathrm{Nil} \mathbf{Z}[T] / T^{n}\right)$ is the image under the connecting homomorphism $\rho_{2}$ of the Steinberg symbol $\left\{X^{-1}, 1-X^{-1} T\right\}$, and where $\theta$ is the pairing

$$
\theta: \mathscr{C}_{\infty} K_{p}(R) \otimes \widetilde{K}_{p}\left(\mathrm{Nil} \mathrm{Z}[T] / T^{n}\right) \rightarrow \mathscr{C}_{n} K_{p+q}(R) .
$$

The maps $\partial^{(n)}$ clearly induce a map

$$
\partial: \mathscr{C}_{\infty} K_{p}(R) \rightarrow \mathscr{C}_{\infty} K_{p+1}(R) .
$$

Proposition 1.5. Let $R$ be any ring. Then the following identities hold for the operations $V_{n}, F_{n}$, and $\partial$ on the group of curves of $K_{*}(R)$.

(1) $V_{n} \circ \partial=n \partial \circ V_{n} ; \partial \circ F_{n}=n F_{n} \circ \partial, \forall n$.

(2) $\bullet$ If $(m, 2)=1$, then $F_{m} \circ \partial \circ V_{m}=\partial$.

(3) - If $R$ is an $\mathbf{F}_{2}$-algebra, then $F_{2} \circ \partial \circ V_{2}=\partial$.

- In all cases, $\left(F_{2} \circ \partial \circ V_{2}-\partial\right) \circ V_{n}=V_{n} \circ\left(F_{2} \circ \partial \circ V_{2}-\partial\right)$.

- $2 \partial^{2}=0$.

(4) - If $R$ is an $\mathbf{F}_{2}$-algebra, then $\partial^{2}=0$.

Proof. As with Proposition 2.1, the idea is to use the nondegeneracy of the pairing with $\widetilde{K}_{*} \operatorname{Nil}(B)$ and Stienstra's identities for operations on $\widetilde{K}_{*} \operatorname{Nil}(B)$. In [St 1], Stienstra defined a derivation $\partial$ on $\widetilde{K}_{*}(\operatorname{Nil}(B))$ for any commutative ring $B$ as follows.

Let $\operatorname{End}(\mathbf{Z}[y] ; x(x-y))$ denote the exact category of pairs $(M, \alpha)$ such that $M$ is a finitely generated projective $\mathbf{Z}[y]$-module and $\alpha$ an endomorphism of $M$ such that $\alpha(\alpha-y)$ is nilpotent. Again, Quillen's proof of the localization theorem of $K$-theory identifies $B Q \operatorname{End}(Z[y] ; x(x-y))$ with the homotopy fiber of the localization

$$
B Q \mathscr{P}(\mathbf{Z}[x, y]) \rightarrow B Q \mathscr{P}\left(\mathbf{Z}\left[x, y, x^{-1},(x-y)^{-1}\right]\right) .
$$

Thus, there is a long exact sequence

$$
\begin{aligned}
\cdots \rightarrow K_{i}(\mathbf{Z}[x, y]) & \rightarrow K_{i}\left(\mathbf{Z}\left[x, y, x^{-1},(x-y)^{-1}\right]\right) \\
& \stackrel{\tau}{\rightarrow} K_{i-1}(\operatorname{End}(\mathbf{Z}[y] ; x(x-y))) \rightarrow \cdots .
\end{aligned}
$$


Now, there is a biexact functor:

$$
\begin{aligned}
\operatorname{End}(\mathbf{Z}[y] ; x(x-y)) \times \operatorname{Nil}(B) & \stackrel{\phi}{\rightarrow} \mathrm{Nil}(B) \\
((M, \alpha),(N, \beta)) & \mapsto\left(M \otimes_{\mathbf{Z}[y]} N_{\beta}, \alpha \otimes 1\right)
\end{aligned}
$$

for any commutative ring $B$. Stienstra defines a derivation

$$
\partial: \widetilde{K}_{p}(\operatorname{Nil}(B)) \rightarrow \widetilde{K}_{p+1}(\operatorname{Nil}(B))
$$

by $\partial(\alpha)=\phi(\tau(\{y-x, x\}), \alpha)$. The following lemma shows how the derivation on curves is related to the derivation on $\widetilde{K}_{*}(\mathrm{Nil}(B))$.

Lemma 1.6. Let $A$ be a ring and let $B$ be a commutative ring. Then, for any $\alpha \in \mathscr{C}_{\infty} K_{p}(A), \beta \in \widetilde{K}_{q}(\mathrm{Nil}(B))$, we have that

$$
\theta(\partial \alpha, \beta)=\theta(\alpha, \partial \beta) \text {. }
$$

Proof. There are multiexact functors:

$$
\begin{aligned}
\mathscr{P}\left(A[T] / T^{m(n+1)}\right) \times \mathrm{Fil}_{m(n+1)}\left(\operatorname{Nil}\left(\mathbf{Z}[y] / y^{n}\right)\right) \times \operatorname{Fil}_{n}(\operatorname{Nil}(B)) \\
\quad \psi_{1}^{m, n} \mathscr{P}\left(A \otimes_{\mathbf{Z}} B\right)
\end{aligned}
$$

and

$$
\begin{aligned}
\mathscr{P}\left(A[T] / T^{m(n+1)}\right) \times \operatorname{Fil}_{m}(\operatorname{End}(\mathbf{Z}[y] ; x(x-y))) \times \operatorname{Fil}_{n}(\operatorname{Nil}(B)) \\
\stackrel{\psi_{2}^{m, n}}{\longrightarrow} \mathscr{P}\left(A \otimes_{\mathbf{Z}} B\right)
\end{aligned}
$$

defined by

$$
\psi_{1}^{m, n}(M,(N, \alpha),(P, \beta))=M \otimes N_{\alpha} \otimes P_{\beta}
$$

and

$$
\psi_{2}^{m, n}(M,(U, \gamma),(P, \beta))=M \otimes\left(U \otimes P_{\beta}\right)_{\gamma \otimes 1} .
$$

There is a natural functor

$$
j^{m, n}: \operatorname{Fil}_{m}(\operatorname{End}(\mathbf{Z}[y] ; x(x-y))) \rightarrow \operatorname{Fil}_{m(n+1)}\left(\operatorname{Nil}\left(\mathbf{Z}[y] / y^{n}\right)\right)
$$

defined by

$$
(M, \alpha) \mapsto\left(M \otimes_{\mathbf{Z}[y]} \mathbf{Z}[y] / y^{n}, \alpha \otimes 1\right) .
$$

Moreover, it is easy to see that the functors $\psi_{2}^{m, n}$ and $\psi_{1}^{m, n} \circ\left(I \times j^{m, n} \times I\right)$ are isomorphic.

Now, let

$$
j^{\infty, n}: K_{1}(\text { End Z }[y] ; x(x-y)) \rightarrow \mathscr{C}_{n} K_{1}(\mathrm{Nil} \mathbf{Z})
$$

be defined by $j^{\infty, n}=\operatorname{colim}_{n} K_{1}\left(j^{m, n}\right)$.

Claim. $j^{\infty, n}(\tau\{y-x, x\})=\rho\left\{x^{-1}, 1-x^{-1} y\right\}$.

To prove the claim, one notes that the commutativity of the diagram:

$$
\begin{aligned}
& K_{1}(\operatorname{End}(\mathbf{Z}[y] ; x(x-y))) \stackrel{\tau}{\longleftarrow} K_{2}\left(\mathbf{Z}\left[x, y, x^{-1},(x-y)^{-1}\right]\right) \\
& \downarrow^{\infty, n} \quad \downarrow-\otimes_{\mathbf{Z}[y]} \mathbf{Z}[y] / y^{n^{n}} \\
& K_{1}\left(\operatorname{Nil}\left(\mathbf{Z}[y] / y^{n}\right)\right) \stackrel{\rho}{\longleftarrow} K_{2}\left(\mathbf{Z}\left[x, y, x^{-1}\right] / y^{n}\right)
\end{aligned}
$$


implies that

$$
\begin{aligned}
j^{\infty, n}(\tau\{y-x, x\}) & =\rho\{y-x, x\}=\rho\left\{-x\left(1-x^{-1} y\right), x\right\} \\
& =\rho\{-x, x\}+\rho\left\{x^{-1}, 1-x^{-1} y\right\} .
\end{aligned}
$$

Since $\{-x, x\}$ is always zero, this proves the claim.

Now, if we take filtered colimits and cofiltered limits in the only reasonable way, we can define

$$
\begin{gathered}
\mathscr{C}_{\infty} K_{p}(A) \times \mathscr{C}_{\infty} \widetilde{K}_{q}(\operatorname{Nil} \mathbf{Z}) \times \widetilde{K}_{r}(\operatorname{Nil}(B)) \stackrel{\psi_{1}^{\infty} \rightarrow \infty}{\rightarrow} K_{p+q+r}(A \otimes B), \\
\mathscr{C}_{\infty} K_{p}(A) \times \widetilde{K}_{q}(\text { End Z }[y] ; x(x-y)) \times \widetilde{K}_{r}(\operatorname{Nil}(B)) \stackrel{\psi_{2}^{\infty, \infty} \rightarrow}{\rightarrow} K_{p+q+r}(A \otimes B) .
\end{gathered}
$$

We still have an isomorphism of functors $\psi_{2}^{\infty, \infty} \cong \psi_{1}^{\infty, \infty} \circ\left(I \times j^{\infty, \infty} \times I\right)$ and equalities:

$$
\begin{gathered}
\psi_{1}^{\infty, \infty}\left(\alpha, \rho\left\{x^{-1}, 1-x^{-1} y\right\}, \beta\right)=\theta(\partial \alpha, \beta) \\
\| \\
\psi_{2}^{\infty, \infty}(\alpha, \tau\{y-x, x\}, \beta)=\theta(\alpha, \partial \beta) .
\end{gathered}
$$

This completes the proof of the lemma.

The results of the proposition now follow from Stienstra's identities ([St 1]) for the derivation on the complex $\widetilde{K}_{*}(\mathrm{Nil}(B))$.

Corollary 1.7. If $S$ is the complement of a set $\mathscr{U}$ of (rational) primes that are invertible in a ring $A$, then the derivation $\partial$ of the complex $\mathscr{C}_{\infty} K_{*}(A)$ commutes with the projection onto the group $\mathscr{T}^{S} \mathscr{C}_{\infty} K_{*}(A)$ of $S$-typical curves on the $K$ theory of $A$.

Corollary 1.8. The derivation $\partial$ on $\mathscr{C}_{\infty} K_{*}(A)$ is a continuous linear map.

Proof. Let $n_{0} \geq 1$ be such that the image of $\widetilde{K}_{1}\left(\operatorname{Fil}_{n_{0}} \operatorname{End}(\mathbf{Z}[y] ; x(x-y))\right)$ in $\widetilde{K}_{1}(\operatorname{End}(Z[y] ; x(x-y)))$ contains the element $\tau\{y-x, x\}$ (i.e., the element used in Steinstra's definition of the derivation on $\left.\widetilde{K}_{*}(\operatorname{Nil}(B))\right)$. Then $\partial$ maps the image of $\widetilde{K}_{*}\left(\operatorname{Fil}_{m} \operatorname{Nil}(B)\right)$ to $\widetilde{K}_{*+1}\left(\operatorname{Fil}_{n_{0}(m+1)} \operatorname{Nil}(B)\right)$. Now by the above lemma, we can translate this into the statement that the derivation on the complex of curves maps $\operatorname{Fil}_{n_{0}(m+1)} \mathscr{C}_{\infty} K_{p}(A)$ to $\operatorname{Fil}_{m} \mathscr{C}_{\infty} K_{p+1}(A)$.

\section{The homology in the case of Q-Algebras}

2.1. Goodwillie's theorem. The calculation of the complex $\left(\mathscr{T}^{\varnothing} \mathscr{C}_{\infty} K_{*}(A), \partial\right)$ for Q-algebras $A$ relies on Goodwillie's theorem [Go 1], which says, in particular, that if $A$ is a $\mathbf{Q}$-algebra and $I$ a nilpotent ideal in $A$, then there is an isomorphism

$$
K_{n}(A, I) \stackrel{\cong}{\longrightarrow} H C_{n-1}(A, I) .
$$


(We are using the fact that $K_{*}(A, I)$ is a Q-module [We 1].) Goodwillie's isomorphism is the composite of maps

$$
K_{n}(A, I) \stackrel{\alpha}{\rightarrow} H C_{n}^{-}(A, I) \stackrel{\beta^{-1}}{\rightarrow} H C_{n-1}(A, I)
$$

where $\mathrm{HC}_{*}^{-}$is the version of cyclic homology introduced by Goodwillie (in [Go 1]) and Hood and Jones (in [HJ]). Note in particular that the map $\alpha$ is the rational relative version of a homomorphism

$$
K_{*}(A) \rightarrow H C_{*}^{-}(A)
$$

which is shown in $[\mathrm{HJ}]$ to be multiplicative.

2.2. Stabilization of curves. Prior to Goodwillie's theorem there was no method for calculating $\mathscr{C}_{\infty} K_{*}(A)$ for any large class of rings, $A$. In [St 2], Stienstra showed that, in contrast to the case of $K_{*}^{\text {sym }}$, the (restriction) maps

$$
\mathscr{C}_{N} K_{3}(A) \rightarrow \mathscr{C}_{n} K_{3}(A) \quad(N \geq n)
$$

are usually not surjective-even for Q-algebras. In light of Goodwillie's theorem, we can easily generalize that part of Stienstra's results to describe the restriction homomorphism for the groups of curves on $K_{p}$ of $\mathbf{Q}$-algebras for any $p$. What we will find is that the general pattern follows that noted by Steinstra in the case of $K_{3}$.

Notation (cf. [Ka], [Bur-O]). We will say that the cyclic homology of a Q-algebra $A$ can be written in quasi-split form as

$$
H C_{*}(A)=V_{*} \otimes \mathbf{Q}[u]+W_{*}
$$

if the above is a direct sum decomposition of $H C_{*}(A)$ as a graded abelian group (in which the degree of $u$ is 2 ) and if the "periodicity operator" $S$ of cyclic homology (cf. [LQ]) annihilates the second summand and acts on the first summand by $S\left(\sum_{i \geq 0} v_{i} \otimes u^{i}\right)=\sum_{i \geq 1} v_{i} \otimes u^{i-1}$.

The cyclic homology of $\mathbf{Q}[T] / T^{n}$ has been computed (by Goodwillie and independently by Masuda and Natsume-cf. [Ka]) to be (in quasi-split form):

$$
\mathbf{Q}[u]+\bigoplus_{m \geq 0} \mathbf{Q}_{[2 m]}^{n-1}
$$

(where the superscript in brackets is the degree). Note that the first term in the decomposition is just $H C_{*}(\mathbf{Q})$. If, then, we denote the quotient of $H C_{*}\left(\mathbf{Q}[T] / T^{n}\right)$ divided by the subgroup $H C_{*}(\mathbf{Q})$ by $\widetilde{H C}_{*}\left(\mathbf{Q}[T] / T^{n}\right)$ (and similarly for Hochschild homology $\widetilde{H H}_{*}$ ), then the "Connes-Gysin sequence" splits into short exact sequences:

$$
0 \rightarrow \widetilde{H C}_{m-1}\left(\mathbf{Q}[T] / T^{n}\right) \stackrel{\widetilde{B}}{\rightarrow} \widetilde{H H}_{m}\left(\mathbf{Q}[T] / T^{n}\right) \stackrel{\widetilde{I}}{\rightarrow} \widetilde{H C}_{m}\left(\mathbf{Q}[T] / T^{n}\right) \rightarrow 0
$$


Now, $H H_{*}\left(\mathbf{Q}[T] / T^{n}\right)$ has been computed (cf., for example, 1.10 of [GRW]) in terms of cycles in the standard resolution. The result is as follows:

$$
H H_{m}\left(\mathbf{Q}[T] / T^{n+1}\right)= \begin{cases}\mathbf{Q}[T] / T^{n+1} & \text { on generator } 1 \text { if } m=0, \\ \mathbf{Q}[T] / T^{n} & \text { on generator } \tau_{n}^{i} u \text { if } m=2 i+1, \\ \mathbf{Q}[T] / T^{n} & \text { on generator } T \tau_{n}^{i} \text { if } m=2 i(>0),\end{cases}
$$

where

$$
\tau_{n}=\operatorname{cls}\left(\left[T^{n} \mid T\right]+T\left[T^{n-1} \mid T\right]+\cdots+T^{n-1}[T \mid T]\right), \quad u=\operatorname{cls}([T]),
$$

and the product is the shuffle product.

From this description it is clear that, if $q>2 n$, then the restriction map

$$
\mathbf{Q}[T] / T^{q+1} \rightarrow \mathbf{Q}[T] / T^{n+1}
$$

induces a map that annihilates $\tau_{q}$ and that, thus, the induced map on Hochschild homology is zero in degrees $>1$.

It follows, then, from the Connes-Gysin short exact sequence that the restriction homomorphism

$$
\widetilde{H C}_{m}\left(\mathbf{Q}[T] / T^{q+1}\right) \rightarrow \widetilde{H C}_{m}\left(\mathbf{Q}[T] / T^{n+1}\right)
$$

is zero if $m>1$. For $m=0$, the restriction is the natural surjection:

$$
\mathrm{Q}[T] / T^{q+1} \rightarrow \mathrm{Q}[T] / T^{n+1} .
$$

For $m=1$, the Connes-Gysin sequence shows that $\widetilde{H C}_{1}\left(\mathbf{Q}[T] / T^{n+1}\right)=0$ for all $n$.

The Künneth formula ([Bur-O] or [Ka]) for cyclic homology then implies that there are natural isomorphisms for any $\mathbf{Q}$-algebra $A$ :

$$
\lim _{\leftarrow n} \widetilde{H C}_{m}\left(A[T] / T^{n+1}\right) \cong \lim _{\leftarrow n}\left(H H_{m}(A) \otimes T \mathbf{Q}[T] / T^{n+1}\right) .
$$

Moreover, the proof of Theorem 5.6 of [GRW] shows that this isomorphism is the limit of the composite maps:

$$
\begin{aligned}
H H_{*}(A) \otimes \widetilde{H H}_{0}\left(\mathbf{Q}[T] / T^{n+1}\right) & \stackrel{\text { shuffle }}{\rightarrow} \widetilde{H H}_{*}\left(A[T] / T^{n+1}\right) \\
& \stackrel{\widetilde{I}}{\rightarrow} \widetilde{H C}{ }_{*}\left(A[T] / T^{n+1}\right) .
\end{aligned}
$$

The isomorphism $\mathscr{C}_{\infty} K_{*+1}(A) \stackrel{\sim}{\rightarrow} H H_{*}(A) \hat{\otimes} T \mathrm{Q}[[T]]$ allows us to define the operations $V_{n}, F_{n}$, and $\partial$ on the right-hand side. In order to calculate the complex of groups of $\varnothing$-typical curves and its homology, we need explicit descriptions of the action of each of these three operators on $H H_{*}(A) \hat{\otimes} T \mathrm{Q}[[T]]$.

The operation $V_{n}$ is (clearly) the homomorphism

$$
\alpha \otimes T^{k} \mapsto \alpha \otimes T^{n k}
$$

For $F_{n}$, we need to examine how the transfer map in $K$-theory gives rise to a map in cyclic homology relative to a nilpotent ideal. 
The map

$$
\begin{aligned}
\phi(n): A[T] / T^{m} & \rightarrow A[T] / T^{n m}, \\
T & \mapsto T^{n}
\end{aligned}
$$

induces a map

$$
\phi(n)_{p}: \mathrm{GL}_{p}\left(A[T] / T^{n m}\right) \rightarrow \mathrm{GL}_{n p}\left(A[T] / T^{m}\right)
$$

with the property that

$$
\operatorname{trace}\left(\phi(n)_{p}(x)\right)=\operatorname{Tr}_{\phi(n)}(\operatorname{trace}(x)) .
$$

Moreover,

$$
\operatorname{Tr}_{\phi(n)}\left(T^{k}\right)= \begin{cases}0 & \text { if } n+k, \\ n T^{k / n} & \text { if } n \mid k .\end{cases}
$$

The Dennis trace map [Ig] $K_{*}(A) \rightarrow H H_{*}(A)$ is the composite of the Hurewicz homomorphism with the map on homology induced by the trace map:

$$
\mathrm{Z} B_{*} \mathrm{GL}(A) \rightarrow \mathscr{H}_{*}(A)
$$

where the right-hand side is the group of Hochschild chains. From the effect of $\phi(n)$ on traces and from the fact that the Dennis map determines Goodwillie's homomorphism, it is easy to see that the action of $F_{n}$ on $H H_{*}(A) \hat{\otimes} T \mathbf{Q}[[T]]$ is given by

$$
\alpha \otimes T^{k} \mapsto \begin{cases}0 & \text { if } n \nmid k, \\ n \alpha \otimes T^{k / n} & \text { if } n \mid k .\end{cases}
$$

Corollary 2.1. For any Q-algebra $A$, there is a natural isomorphism:

$$
\mathscr{T}^{\varnothing} \mathscr{C}_{\infty} K_{*+1} \cong H H_{*}(A) \text {. }
$$

The remainder of the paper is devoted to the calculation of the map $\delta$ that makes the following diagram commutative:

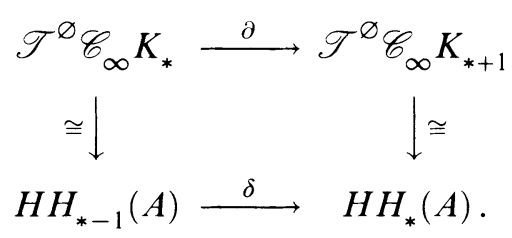

I do not know how to do so in full generality, but only when the Q-algebra $A$ satisfies the " $K$-theoretic curves conditions" below.

$K$-theoretic curves conditions: A ring $A$ is said to satisfy the $K$-theoretic curves condition if the natural map

$$
\widetilde{K}_{n}(A[[T]]) \rightarrow \mathscr{C}_{\infty} K_{n}(A)
$$

is a surjection for every $n \geq 0$. 


\section{Examples.}

(1) If $A$ is a smooth commutative algebra over $\mathbf{Q}$, then $A$ satisfies the $K$-theoretic curves condition. Indeed, $H H_{*}(A)$ is naturally isomorphic to the exterior algebra $\Omega_{A}^{*}$ and, for all $n, \widetilde{K}_{2}\left(A[T] / T^{n}\right)\left(\cong \Omega_{A}^{1} \otimes T \mathbf{Q}[t] / T^{n}\right)$ is generated by Dennis-Stein symbols of the form $\left\langle a T^{k}, b\right\rangle[\mathrm{MS}]$ which are obviously in the image of the restriction map from $\widetilde{K}_{2}(A[[T]])$. The multiplicativity of Goodwillie's map then shows that

$$
\widetilde{K}_{m+1}(A[[T]]) \rightarrow \Omega_{A}^{m} \hat{\otimes} \mathbf{Q}[[T]]
$$

is surjective for all $m \geq 0$.

(2) If $A$ satisfies the $K$-theoretic curves condition, then so does the matrix ring $\mathscr{M}_{n}(A)$ for any $n$.

Suppose, now, that $A$ is a $\mathbf{Q}$-algebra satisfying the $K$-theoretic curves condition. We first define a (coherent) set of maps for each $n$ :

$$
d_{n}: K_{*}(A[[T]]) \rightarrow K_{*+1}\left(A[T] / T^{n}\right)
$$

and a pairing:

$$
\tilde{\theta}: K_{p}(A[[T]]) \times K_{q}(\operatorname{Nil}(B)) \rightarrow K_{p+q}(A \otimes B)
$$

(for commutative $\mathbf{Q}$-algebras $B$ ) such that the following diagram commutes:

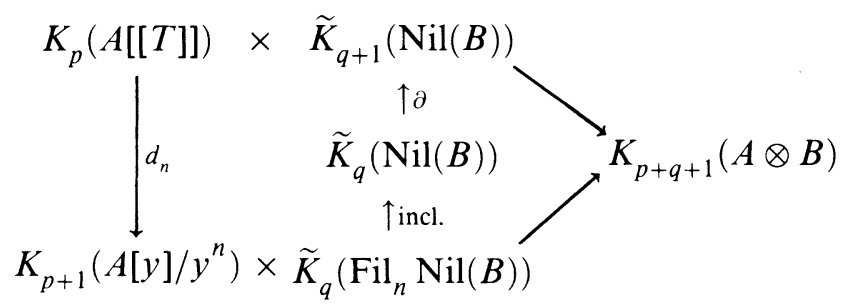

The pairing $\widetilde{\theta}$ is the map on $K$-theory induced by the biexact functor:

$$
\begin{aligned}
\mathscr{P}(A[[T]]) \times \mathrm{Nil}(B) & \rightarrow \mathscr{P}(A \otimes B), \\
(M,(N, \beta)) & \mapsto M \otimes_{\mathrm{Z}[[T]]} N_{\beta} .
\end{aligned}
$$

The map $d_{n}$ is defined as follows. The biexact functor

$$
\begin{aligned}
\mathscr{P}(A[[T]]) \times \operatorname{Nil}\left(\mathbf{Z}[y] / y^{n}\right) & \rightarrow \operatorname{Nil}\left(A[y] / y^{n}\right), \\
(M,(N, \beta)) & \mapsto\left(M \otimes_{\mathbf{Z}[[T]]} N_{\beta}, T\right)
\end{aligned}
$$

induces a map of homotopy fibrations:

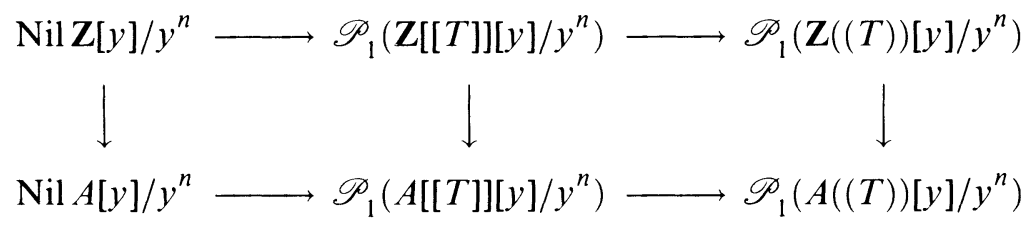

where $A((T))$ denotes $A[[T]]\left[T^{-1}\right]$. 
We now define

$$
d_{n}: K_{p}(A[[T]]) \rightarrow K_{p+1}\left(A[y] / y^{n}\right)
$$

to be the composite

$$
K_{p}(A[[T]]) \stackrel{(1)}{\rightarrow} K_{p+1}\left(\operatorname{Nil}\left(A[y] / y^{n}\right)\right) \stackrel{(2)}{\rightarrow} K_{p+1}\left(A[y] / y^{n}\right)
$$

in which the map (1) is the pairing with the element $\rho\left\{T^{-1}, 1-T^{-1} y\right\}$ of $K_{1}\left(\mathrm{Nil} \mathrm{Z}[y] / y^{n}\right)$ (and $\rho$ the connecting homomorphism in the homotopy sequence of the above fibration) and (2) is the map on $K$-theory induced by the "forgetful" map Nil(?) $\rightarrow \mathscr{P}(?)$.

Now, recall Steinstra's definition of the derivation $\partial$ of the complex $\widetilde{K}_{*}(\operatorname{Nil}(B))$ (cf. the proof of Proposition 2.5 above), via multiplication by the element $\tau\{y-x, x\}$ of $K_{1}($ End $\mathbf{Z}[y] ; x(x-y))$. There is an alternative way of defining Steinstra's derivation as follows. First, there is a biexact functor

$$
\begin{aligned}
\operatorname{Nil} Z[y] / y^{n} \times \operatorname{Fil}_{n} \operatorname{Nil}(B) & \rightarrow \operatorname{Nil}(B), \\
((U, \alpha),(N, \beta)) & \mapsto\left(U \otimes_{\mathrm{Z}[y] / y^{n}} N_{\beta}, \alpha \otimes 1\right) .
\end{aligned}
$$

Now, there is an exact functor:

$$
\begin{aligned}
\nu: \operatorname{End}(\mathbf{Z}[y] ; x(x-y)) & \rightarrow \operatorname{Nil~Z}[y] / y^{n} \\
(M, \rho) & \mapsto\left(M \otimes_{\mathbf{Z}[y]} \mathbf{Z}[y] / y^{n}, \rho \otimes 1\right)
\end{aligned}
$$

which induces a map of fibrations:

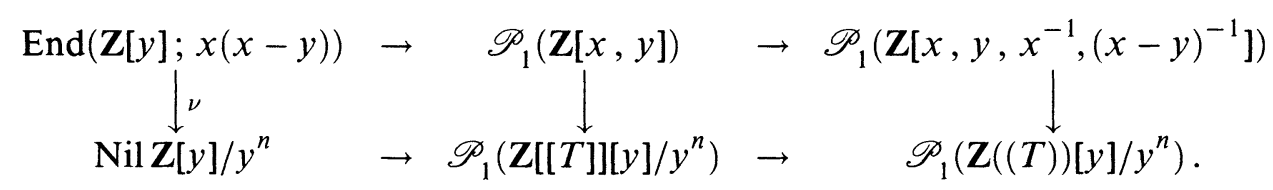

Note that $K_{1}(\nu)$ maps $\tau\{y-x, x\}$ to $\rho\left\{T^{-1}, 1-T^{-1} y\right\}$. We can therefore define $\partial$ on $\widetilde{K}_{*}(\mathrm{Nil} B)$ in terms of the pairing of $\widetilde{K}_{*}\left(\mathrm{Fil}_{n}(\mathrm{Nil} B)\right)$ with the element $\rho\left\{T^{-1}, 1-T^{-1} y\right\}$ of $\mathrm{Nil} \mathbf{Z}[y] / y^{n}$. The commutativity of $(\dagger)$ now follows easily.

Before we can express $\partial$ in terms of a map on Hochschild homology groups, we need additional results on cyclic homology, which are the subject of the next section.

2.3. A completed cone over a ring. The main difficulty remaining in the computation of the homology of the complex $\left(\mathscr{T}^{\varnothing} \mathscr{C}_{\infty} K_{*}(A), \partial\right)$ is how to translate $\partial$ (defined in terms of the $K$-theory of a category $\mathrm{Nil}(B)$ ) into a map on relative cyclic homology. To accomplish this, we modify an argument used in Quillen's proof of the "fundamental theorem" of $K$-theory [GQ]. This involves a sort of completion of the "cone" of a ring.

For any ring $R$, we define rings $\widehat{C}(R)$ and $\widehat{S}(R)$ as follows. 
Definition. $\widehat{C}(R)$ is the ring of row-finite matrices with entries in $R$, i.e., matrices $X=\left(x_{i, j}\right)_{i, j \in \mathbf{N}}$ such that $x_{i, j} \in R$ and such that, for each $i \in \mathbf{N}$, $\exists j(X, i) \in \mathbf{N}$ such that $x_{i, j}=0, \forall j>j(X, i)$.

Let $\lambda(R)$ be the two-sided ideal of "uniformly row-finite" matrices in $X=$ $\left(x_{i, j}\right) \in \widehat{C}(R)$ such that $\exists j(X) \in \mathbf{N}$ with the property that $x_{i, j}=0, \forall j>$ $j(X)$.

We denote the quotient $\widehat{C}(R) / \lambda(R)$ by $\widehat{S}(R)$.

Note that the ring $\lambda(R)$ does not satisfy Wagoner's condition $(*)$ [Wa]-i.e., does not have "local two-sided identity elements." This means that there are problems of excision in defining $K_{*}(\lambda(R))$. We do, however, have the following commutative diagram in which $F$ is the homotopy fiber of the map

$$
B Q \mathscr{P}(\widehat{C}(R)) \rightarrow B Q \mathscr{P}(\widehat{S}(R))
$$

and in which all the rows are homotopy-fibrations (cf. [GQ]).

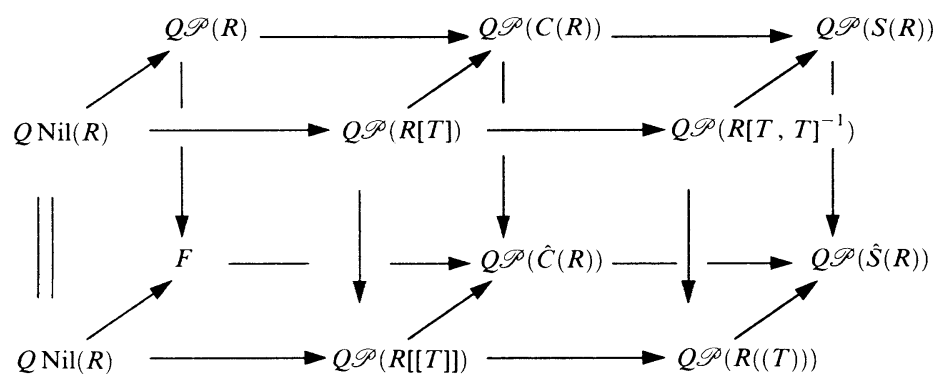

The commutativity of the front face and the identification of $Q \operatorname{Nil}(R)$ as the homotopy fiber of

$$
Q \mathscr{P}(R[[T]]) \rightarrow Q \mathscr{P}(R((T)))
$$

—where $R((T))$ denotes $R[[T]]\left[T^{-1}\right]$-are a result of Karoubi (cf. [We 2]). The arrow $Q \mathscr{P}(R[[T]]) \rightarrow Q \mathscr{P}(\widehat{C}(R))$ comes from the homomorphism of $R$ algebras:

$$
\begin{aligned}
& R[[T]] \rightarrow \widehat{C}(R) \\
& T \mapsto \tau=\left[\begin{array}{ccccc}
0 & \cdots & & \\
1 & 0 & & \\
0 & 1 & 0 & \\
\vdots & 0 & 1 & 0 \\
& \vdots & & \ddots
\end{array}\right] .
\end{aligned}
$$

The diagram makes evident the result of the following lemma. 
Lemma 2.2. For any ring $R$, there is a commutative diagram (natural in $R$ ):

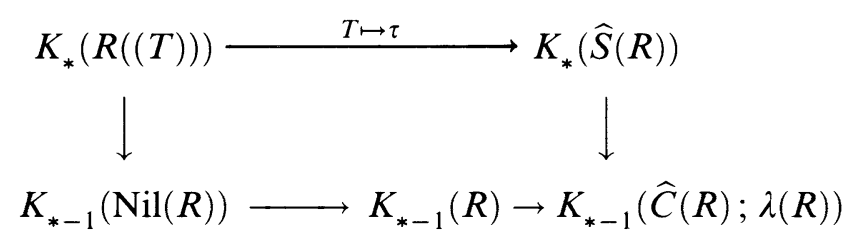

where $K_{*-1}(\widehat{C}(R) ; \lambda(R))=\pi_{*}(F)$ ( $F$ as in the previous diagram) and where the left map is the connecting homomorphism of the homotopy fibration:

$$
Q \operatorname{Nil}(R) \rightarrow Q \mathscr{P}_{1}(R[[T]]) \rightarrow Q \mathscr{P}_{1}(R((T))) .
$$

In fact, the right-hand arrow in the diagram of the lemma is an isomorphism. This follows from the following results.

Lemma 2.3. For any ring $R, \widehat{C}(R)$ is an infinite sum ring.

Proof. The terminology "infinite sum ring" comes from [Wa], and the proof that $\widehat{C}(R)$ is an infinite sum ring is a simple modification of Wagoner's result for $C(R)$ : one simply replaces consideration of "locally-finite endomorphisms of $\coprod_{\mathrm{N}} R$ " by "continuous endomorphisms of $\prod_{\mathrm{N}} R$."

Corollary 2.4. $K_{n}(\widehat{C}(R))=0$ for all $n \geq 0$.

This is a consequence of Wagoner's result [Wa] that $K_{n}(A)=0$ for all infinite sum rings $A$.

Corollary 2.5. The diagram of Lemma 3.2 determines a commutative diagram for any ring $R$ :

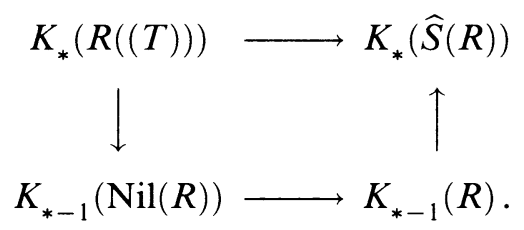

Before we can apply Goodwillie's isomorphism, we need to discuss the cyclic homology of $\widehat{C}(R)$ for Q-algebras $R$.

Proposition 2.6. For any Q-algebra $R$ and any $n \geq 0$

$$
H C_{n}(\widehat{C}(R))=0 \text {. }
$$

Proof. The idea is to mimic the proof of the equivalent result for $K$-theorythat is, to use the fact that $\widehat{C}(R)$ is an infinite sum ring to prove the acyclicity of the Lie algebra $\operatorname{gl}(\widehat{C}(R))$.

For any infinite sum ring $A$ and any $x=\left(x_{i, j}\right), y=\left(y_{i, j}\right) \in \operatorname{gl}(A)$, we define

$$
x \oplus y=\left(x_{i, j} \oplus y_{i, j}\right)
$$

where the $\oplus$ on the right-hand side is the direct sum map $A \times A \rightarrow A$. Note that, as in Wagoner's proof of the acyclicity of $\mathrm{GL}(A)$ [Wa], for any finite 
subset $x_{1}, \ldots, x_{n}$ of $\operatorname{gl}(A)$, there exist elements $c, d$ of $\mathrm{GL}(A)$ such that

$$
\begin{aligned}
c\left(x_{i} \oplus 0\right) c^{-1} & =x_{i}, \\
d\left(0 \oplus x_{i}\right) d^{-1} & =x_{i} \text { for } i=1, \ldots, n .
\end{aligned}
$$

Again, following Wagoner's argument, we define $\tau: \operatorname{gl}(A) \rightarrow \operatorname{gl}(A)$ by $\tau\left(\left(x_{i, j}\right)\right)=\left(x_{i, j}^{\infty}\right)$, where $x \mapsto x^{\infty}$ is the infinite sum endomorphism of $A$. This satisfies the condition

$$
x \oplus \tau(x)=\tau(x) \quad \forall x \in \operatorname{gl}(A) .
$$

From this, it is easy to prove that the rational Lie algebra homology $H_{*}(\operatorname{gl}(A))$ is zero in positive degrees, as follows.

Let $L$ denote $\operatorname{gl}(A)$, and let $\Delta$ denote the diagonal map

$$
\begin{aligned}
\Delta: L & \rightarrow L \times L, \\
x & \mapsto(x, x) .
\end{aligned}
$$

The equality ( $\ddagger)$ shows that the endomorphisms $\oplus \circ(I \times \tau) \circ \Delta$ and $\tau$ agree on $L$.

$\Delta$ defines a coalgebra structure on $H_{*}(L)$. There is also a multiplication:

$$
\tilde{\oplus}: H_{*}(L) \otimes_{\mathbf{Q}} H_{*}(L) \stackrel{\cong}{\rightrightarrows} H_{*}(L \times L) \stackrel{H_{*}(\oplus)}{\rightarrow} H_{*}(L) .
$$

Claim. If we denote by [1] the homology class of the cycle $1 \in \mathbf{Q}$ in the Koszul resolution for $L$, then [1] is the unit for the multiplication $\tilde{\oplus}$.

To prove this claim, suppose $z \in \Lambda^{n}(L)$. Then, $[1] \tilde{\oplus}[z]=[0 \oplus z]$. Now, by (†), $0 \oplus z$ is $\mathrm{GL}(A)$-conjugate to $z$; however, $\mathrm{GL}(A)$ acts trivially on $H_{*}(L)$ (this follows, for example, from Goodwillie's argument, pp. 402-403 of [Go 2]). Thus, $[0 \oplus z]=[z]$. Similarly $[z] \tilde{\oplus}[1]=[z]$, proving the claim.

Since $H_{*}(L)$ is, thus, a bialgebra, in order to prove that it is zero in positive degrees, it suffices to prove that there are nonzero primitive elements. This follows from the equality of $\oplus \circ(I \times \tau) \circ \Delta$ and $\tau$, for if $[z] \in H_{*}(L)$ is a primitive element, then

$$
\begin{aligned}
H_{*}(\tau)([z]) & =H_{*}(\oplus \circ(I \times \tau) \circ \Delta)([z]) \\
& =H_{*}(\oplus \circ(I \times \tau))([z] \otimes[1]+[1] \otimes[z]) \\
& =[z]+H_{*}(\tau)([z]) .
\end{aligned}
$$

This proves that $H_{*}(\mathrm{gl}(A))$ is zero in positive degrees for any infinite sum ring and Q-algebra $A$. It follows from [LQ] that the cyclic homology of $A$ is zero in all degrees.

The inclusion of (nonunital) Q-algebras $\lambda(R) \rightarrow \widehat{C}(R)$ induces a long exact sequence in cyclic homology:

$$
\begin{aligned}
\cdots & \rightarrow H C_{n}(\lambda(R)) \rightarrow H C_{n}(\widehat{C}(R)) \rightarrow H C_{n}(\widehat{C}(R) ; \lambda(R)) \\
& \stackrel{\delta_{n}}{\rightarrow} H C_{n-1}(\lambda(R)) \rightarrow \cdots .
\end{aligned}
$$


The projection $\widehat{C}(R) \rightarrow \widehat{S}(R)$ induces maps:

$$
\pi_{n}: H C_{n}(\widehat{C}(R) ; \lambda(R)) \rightarrow H C_{n}(\widehat{S}(R))
$$

Since $H C_{n}(\widehat{C}(R))=0$ for all $n$, there is, thus, a homology suspension map:

$$
\sigma_{n-1}=\pi_{n} \circ \delta_{n}^{-1}: H C_{n-1}(\lambda(R)) \rightarrow H C_{n}(\widehat{S}(R))
$$

Let us denote by $\bar{\sigma}_{n-1}$ the composite:

$$
H C_{n-1}(R) \rightarrow H C_{n-1}(\lambda(R)) \stackrel{\sigma_{n-1}}{\rightarrow} H C_{n}(\widehat{S}(R))
$$

The suspension can be described in terms of chains (cf. $\S 2$, No. 6-7 of [Bo]): given a cycle $y$ in $\mathscr{B}(\lambda(R))_{n-1}$ (where $\mathscr{B}(?)_{*}$ is the total complex whose homology is $\left.H C_{*}(?)\right)$, there is a chain $z$ in $\mathscr{B}(\widehat{C}(R))_{n}$ such that $y=\delta z$. If $z$ maps onto the cycle $\bar{z}$ in $\mathscr{B}(\widehat{S}(R))_{n}$, then $\sigma_{n-1}$ maps the homology class of $y$ to the class of $\bar{z}$.

Lemma 2.7. For any Q-algebra, $A$, there is a commutative diagram:

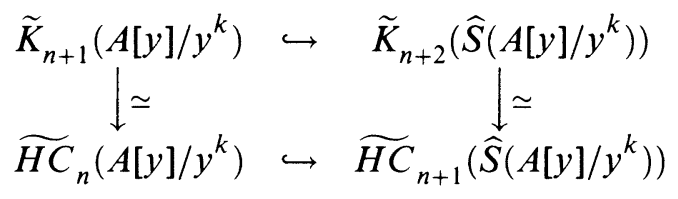

(in which the vertical maps are Goodwillie's isomorphisms, and in which the upper horizontal map is the map of Corollary 2.5). Moreover, the horizontal arrows are injections.

Proof. The commutativity follows easily from the fact that the GoodwillieHood-Jones map for any Q-algebra, $R$ :

$$
\alpha: K_{*}(R) \rightarrow H C_{*}^{-}(R)
$$

is the map on homotopy groups that is induced by a map of spaces:

$$
\tilde{\alpha}: \mathrm{BGL}^{+}(R) \rightarrow X\left(\mathscr{B}_{*}^{-}(R)\right)
$$

in which $\mathscr{B}_{*}^{-}$is the total complex computing $H C_{*}^{-}$and $X$ is the functor from chain complexes to topological spaces which associates with a chain complex $\mathscr{B}_{*}$. the geometric realization of the simplicial abelian group corresponding (via the Dold-Kan equivalence) with the positive chain complex obtained by deleting the terms of negative degree in $\mathscr{B}_{*}$ (see [We 3]). There is, thus, a commutative 
diagram in which the rows are homotopy fibrations:

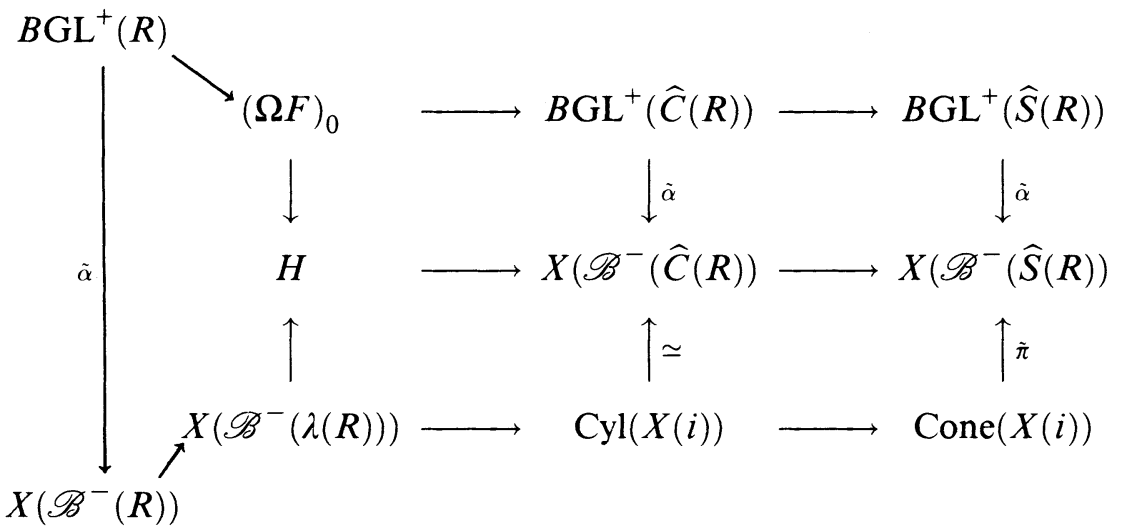

(Here, $H$ is the homotopy fiber of the map $X\left(\mathscr{B}^{-}(\widehat{C}(R))\right) \rightarrow X\left(\mathscr{B}^{-}(\widehat{S}(R))\right)$, $F$ is as in the diagram before Lemma 2.2 and $i$ is the inclusion $\mathscr{B}^{-}(\lambda(R)) \rightarrow$ $\mathscr{B}^{-}(\widehat{C}(R))$.) The homology suspension

$$
H C_{n-1}^{-}(\lambda(R)) \rightarrow H C^{-}(\widehat{S}(R))
$$

is the composite of the map on homotopy groups induced by $\tilde{\pi}$ following the inverse of the connecting homomorphism in the bottommost fibration in the above diagram.

It remains to prove the injectivity of $\widetilde{H C}_{n}\left(A[y] / y^{k}\right) \rightarrow \widetilde{H C}_{n+1}\left(\widehat{S}\left(A[y] / y^{k}\right)\right)$. This follows from the observations that:

(1) Goodwillie's isomorphism and the isomorphism $K_{*}(R) \cong K_{*+1}(S(R))$ (where $S(R)$ is the suspension of a ring $R$ ) prove that $\widetilde{H C}_{*}\left(A[y] / y^{k}\right)$ is isomorphic to $\widetilde{H C}_{*+1}\left(S\left(A[y] / y^{k}\right)\right)$;

(2) the inclusion $S(R) \hookrightarrow \widehat{S}(R)$ induces an injection in cyclic homology for any $\mathbf{Q}$-algebra, $R$.

To prove the second of these reduces to showing that if $z$ is a normalized Hochschild $n$-chain for $S(R)$ that is the boundary of a (normalized) $n+1$ chain for $\widehat{S}(R)$, then there is a (normalized) $n+1$-chain for $S(R)$ whose boundary is also $z$. This in turn reduces to the following (which is easily proved), namely that given a finite set of matrices $X_{1}, \ldots, X_{p}$ in $\widehat{C}(R)$ and a sequence $\left\{m_{n}: n \geq 1\right\}$ of nonnegative integers, there is a sequence $\left\{\tilde{m}_{n}: n \geq 1\right\}$ such that, for every $i, j=1, \ldots, p$,

$$
\pi\left(m_{*}\right)\left(X_{i} X_{j}\right)=\pi\left(m_{*}\right)\left(\pi\left(\tilde{m}_{*}\right) X_{i} \pi\left(\tilde{m}_{*}\right) X_{j}\right)
$$

where $\pi\left(m_{*}\right)$ denotes the linear projection from $\widehat{C}(R)$ onto the subspace of matrices whose $n$th column is zero after the $m_{n}$ th term, for all $n$, that is defined by

$$
\pi\left(m_{*}\right)(X)_{i, j}= \begin{cases}X_{i, j} & \text { if } i \leq m_{j} \\ 0 & \text { if } i>m_{j}\end{cases}
$$


2.4. Calculation of the homology. We can now complete the calculation of the homology of the complex $\left(\mathscr{T}^{\varnothing} \mathscr{C}_{\infty} K_{*}(A), \partial\right)$ for Q-algebras $A$ that satisfy the " $K$-theoretic curves condition."

Recall that we have defined a coherent system of maps:

$$
d_{n}: \widetilde{K}_{*}(A[[T]]) \rightarrow \widetilde{K}_{*+1}\left(A[y] / y^{n}\right)
$$

and an inclusion

$$
j: \widetilde{K}_{*+1}\left(A[y] / y^{n}\right) \hookrightarrow \widetilde{K}_{*+2}\left(\widehat{S}\left(A[y] / y^{n}\right)\right)
$$

such that $j \circ d_{n}$ is the composite:

$$
\widetilde{K}_{*}(A[[T]]) \stackrel{(\mathrm{i})}{\rightarrow} \widetilde{K}_{*+2}\left(A((T))[y] / y^{n}\right) \stackrel{(\mathrm{ii})}{\rightarrow} \widetilde{K}_{*+2}\left(\widehat{S}\left(A[y] / y^{n}\right)\right)
$$

where (i) is the $K$-theory product with the Steinberg symbol $\left\{T^{-1}, 1-T^{-1} y\right\} \in$ $\widetilde{K}_{2}\left(\mathbf{Q}((T))[y] / y^{n}\right)$ and (ii) is the map induced by the $A[y] / y^{n}$-algebra homomorphism

$$
\begin{aligned}
A((T))[y] / y^{n} & \rightarrow \widehat{S}\left(A[y] / y^{n}\right), \\
T & \mapsto \tau .
\end{aligned}
$$

Since the Goodwillie-Hood-Jones map

$$
\alpha: K_{*}(R) \rightarrow H C_{*}^{-}(R)
$$

is multiplicative, the map on $\mathrm{HC}^{-}$corresponding to the homomorphism (i), above, is the cyclic homology product with

$$
\alpha\left(T^{-1}\right) \cdot \alpha\left(1-T^{-1} y\right) \in \widetilde{H C}_{2}^{-}\left(\mathbf{Q}((T))[y] / y^{n}\right) .
$$

Now, the commutativity of the diagram

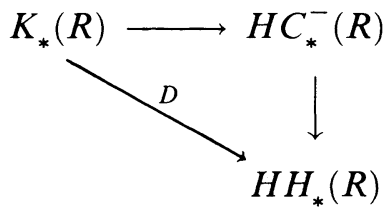

(where $D$ is the Dennis trace) together with the well-known (and easy) fact that $D(\{x\})=\operatorname{cls}\left(x^{-1} \otimes x\right.$ ) (for any unit $x$ in a commutative $\mathbf{Q}$-algebra, $R$ ) shows that

$$
\begin{array}{r}
\alpha\left(T^{-1}\right) \cdot \alpha\left(1-T^{-1} y\right)=\operatorname{cls}\{( \\
\left.\left(1-T^{-1} y\right)^{-1} \otimes T^{-1} \otimes\left(1-T^{-1} y\right)\right) \\
-\left(T\left(1-T^{-1} y\right)^{-1} \otimes\left(1-T^{-1} y\right) \otimes T^{-1}\right) \\
+ \text { higher order terms }\} .
\end{array}
$$

Since, by Goodwillie's theorem, we know that this is in the image of the map

$$
\beta: \widetilde{H C}_{1}\left(\mathbf{Q}((T))[y] / y^{n}\right) \rightarrow \widetilde{H C}_{2}^{-}\left(\mathbf{Q}((T))[y] / y^{n}\right),
$$

the higher order terms must be negligible. 
Now, if we assume that $n=2$, then the Hochschild cycles

$$
\left(T\left(1-T^{-1} y\right)^{-1} \otimes T^{-1} \otimes\left(1-T^{-1} y\right)\right)-\left(T\left(1-T^{-1} y\right)^{-1} \otimes\left(1-T^{-1} y\right) \otimes T^{-1}\right)
$$

and

$$
\left(1 \otimes y \otimes T^{-1}-1 \otimes T^{-1} \otimes y\right)
$$

are homologous. (Their difference is the boundary of the chain

$$
\begin{aligned}
(T & \left.\otimes T^{-1} \otimes y \otimes T^{-1}\right)+\frac{1}{2}\left(1 \otimes T^{-1} \otimes T^{-1} y \otimes y\right) \\
& -\frac{1}{2}\left(1 \otimes T^{-1} y \otimes T^{-1} \otimes y\right)-\frac{1}{2}\left(1 \otimes y \otimes T^{-1} \otimes T^{-1} y\right) \\
& \left.+\frac{1}{2}\left(1 \otimes y \otimes T^{-1} y \otimes T^{-1}\right)+\frac{1}{2}\left(T^{-1} \otimes y \otimes T^{-1} \otimes y\right) .\right)
\end{aligned}
$$

Thus, for $n=2,\left(\beta^{-1} \circ \alpha\right)\left(\left\{T^{-1}, 1-T^{-1} y\right\}\right)$ is the cyclic homology class of $y \otimes T^{-1}$.

Now, we define a map

$$
D_{n}: \widetilde{H C}_{*}(A[[T]]) \rightarrow \widetilde{H C}_{*+2}\left(\widehat{S}\left(A[y] / y^{n}\right)\right)
$$

to be the following composite:

$$
\widetilde{H C}_{*}(A[[T]]) \stackrel{(\mathrm{i})}{\rightarrow} \widetilde{H C}_{*+2}\left(A((T))[y] / y^{n}\right) \stackrel{(\mathrm{ii})}{\rightarrow} \widetilde{H C}_{*+2}\left(\widehat{S}\left(A[y] / y^{n}\right)\right)
$$

where (i) is the (cyclic) homology product with the element $\left(\beta^{-1} \circ \alpha\right)$. $\left(\left\{T^{-1}, 1-T^{-1} y\right\}\right)$, and (ii) is induced by the ring homomorphism $T \mapsto \tau$.

We then have the following commutative diagram:

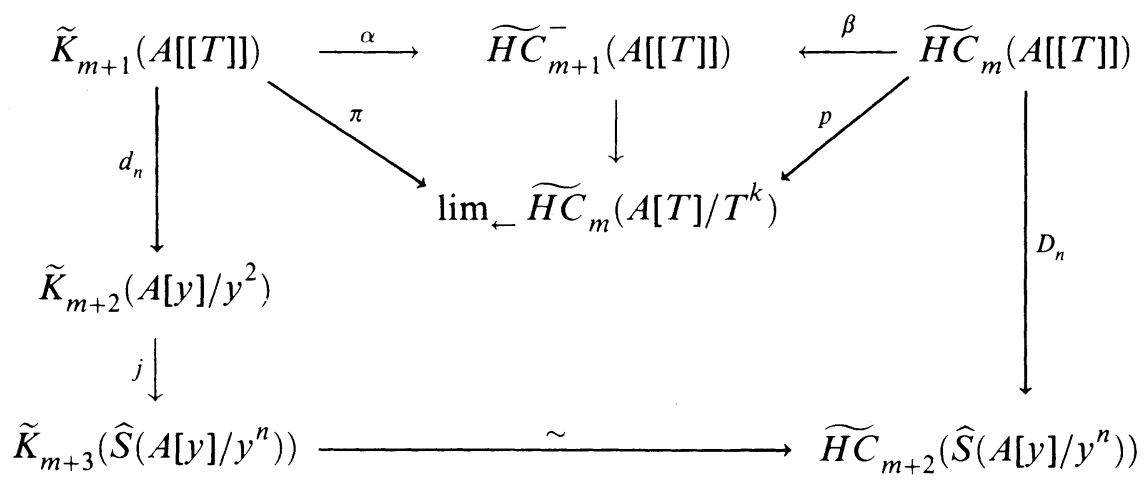

where $\pi$ and $p$ are surjections, $j$ is an injection, and the maps $d_{n}$ induce, in the limit, a map $d_{\infty}$ making a commutative diagram:

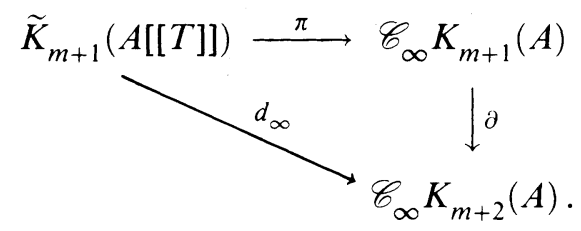


Passing to $\varnothing$-typical curves, we get the following commutative diagram:

$$
\begin{aligned}
\mathscr{T}^{\varnothing} \mathscr{C}_{\infty} K_{*}(A) \simeq & H H_{*-1}(A) \stackrel{\widetilde{J}}{\hookrightarrow} \widetilde{H C}_{*-1}(A[[T]]) \\
\mathscr{T}^{\varnothing} \mathscr{C}_{\infty} K_{*+1}(A) \simeq & \begin{array}{c}
H H_{*}(A) \\
\downarrow \uparrow
\end{array} \\
& \widetilde{H C}_{*}\left(A[y] / y^{2}\right) \hookrightarrow \widetilde{H C}\left(\widehat{S}\left(A[y] / y^{2}\right)\right) .
\end{aligned}
$$

The map $\widetilde{J}$ is the composite

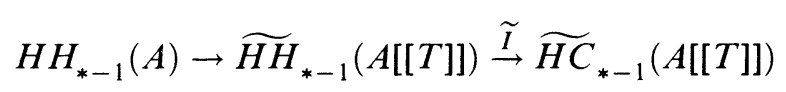

in which the first arrow is the map $\alpha \mapsto \alpha \# T$ (where \# denotes the shuffle product on Hochschild homology, and $T$ is viewed as an element of $\left.\widetilde{H H}_{0}(\mathbf{Q}[[T]])\right)$.

Now, if $x \in H H_{m}(A)$, we calculate $D_{2}(\widetilde{J}(x))$. By Corollary 3.8 and formula 3.2 of [LQ],

$$
\begin{aligned}
D_{2}(\widetilde{J}(x)) & =\phi\left(\operatorname{cls}\left(y \otimes T^{-1}\right) * \widetilde{I}(x \# T)\right) \\
& =\phi \circ I\left\{\operatorname{cls}\left(y \otimes T^{-1}\right) \# B(x \# T)\right\}
\end{aligned}
$$

where $\phi$ is the map on homology induced by the homomorphism of rings $A((T))[y] / y^{2} \rightarrow \widehat{S}\left(A[y] / y^{2}\right)$ and where $*$ is the product in cyclic homology.

If $x$ is the class of the Hochschild chain $x_{0} \otimes \cdots \otimes x_{m}$, this means that

$$
D_{2}(\widetilde{J}(x))=\phi\left(\operatorname{cls}\left\{\sum_{i, j}(-1)^{i m+j}\left(y \otimes x_{i} \otimes \cdots \otimes T^{-1} \otimes \cdots \otimes x_{i-1}\right\}\right)\right.
$$

where the $T^{-1}$ occurs in the $j+1$ position and the entry in the $m-i+2$ position is $T x_{0}$.

Let $z$ be the Hochschild chain for the ring $\widehat{C}\left(A[y] / y^{2}\right)$ defined by

$$
z=\sum_{i, j}(-1)^{i m+j}\left(y \otimes x_{i} \otimes \cdots \otimes \sigma \otimes \cdots \otimes x_{i-1}\right)
$$

where the $x_{i}$ 's are scalar matrices, the $m-i+2$ term in the sum is $\tau x_{0}$, and

$$
\sigma=\left[\begin{array}{ccccc}
0 & 1 & 0 & \cdots & \\
& 0 & 1 & 0 & \cdots \\
& & 0 & 1 & 0 \\
& & & & \ddots
\end{array}\right]
$$

If $b$ denotes the Hochschild boundary, then $b z$ is a sum of commutators, the only nonzero elements of which are

$$
\sum_{i}(-1)^{i m+1}\left(y \otimes x_{i} \otimes \cdots \otimes x_{m} \otimes e x_{0} \otimes \cdots \otimes x_{i-1}\right)
$$

where $e=\sigma \tau-\tau \sigma$ (that is, the matrix having 1 in the upper left corner and zeros elsewhere). 
For convenience, we shorten $A[y] / y^{2}$ to $R$ for the rest of the calculation. Let $\widehat{\Delta}(R)$ denote the subring of diagonal matrices in $\widehat{C}(R)$. Note that $e$ is a central idempotent in $\widehat{\Delta}(R)$ and that $b z$ is a chain in the Hochschild complex $\mathscr{H}_{*}(\widehat{\Delta}(R)) . \mathscr{H}_{*}(\widehat{\Delta}(R))$ is the direct sum of three subcomplexes: $\mathscr{H}_{*}(e \widehat{\Delta}(R))$, $\mathscr{H}_{*}((1-e) \widehat{\Delta}(R))$, and the complex of "mixed" terms, $\mathscr{H}_{*}\left(\widehat{\Delta}(R)_{\text {mix }}\right)$. Since the projection onto the sum of the first two of these induces an isomorphism on homology, $\mathscr{H}_{*}\left(\widehat{\Delta}(R)_{\text {mix }}\right)$ is acyclic. It is easy to see that the element $b z$ of $\mathscr{H}_{*}(\widehat{\Delta}(R))$ can be written in the form

$$
\sum_{i}(-1)^{i m+1}\left(e y \otimes e x_{i} \otimes \cdots \otimes e x_{m} \otimes e x_{0} \cdots e x_{i-1}\right)+v
$$

where $v \in \mathscr{H}_{*}\left(\widehat{\Delta}(R)_{\text {mix }}\right)$. Moreover, the first of these terms is a cycle (since it is just $B x \# y)$. Thus $v$ is a boundary of the subcomplex $\mathscr{H}_{*}(\widehat{\Delta}(R))$-say $v=b u$. Then $b(z-u)=B x \# y$ and the homology class of the image of $z-u$ in $\mathscr{H}_{*}(\widehat{S}(R))$ is the element $D_{2}(\widetilde{J}(x))$. It follows that the suspension homomorphism

$$
\widetilde{H C}_{*}\left(A[y] / y^{2}\right) \rightarrow \widetilde{H C}_{*+1}\left(\widehat{S}\left(A[y] / y^{2}\right)\right)
$$

maps $\operatorname{cls}(B x \# y)$ to $D_{2}(\widetilde{J}(x))$.

We have therefore proved the following result.

Theorem 2.8. Let $A$ be a Q-algebra that satisfies the $K$-theoretic curves condition. Then there is an isomorphism of complexes from the complex $\left(\mathscr{T}^{\varnothing} \mathscr{C}_{\infty} K_{*+1}(A), \partial\right)$ to the complex $\left(H H_{*}(A), B\right)$, where $B$ is Connes' operator on the Hochschild homology groups of $A$.

\section{REFERENCES}

[BI] S. Bloch, Algebraic $K$-theory and crystalline cohomology, Inst. Hautes Études Sci. Publ. Math. 47 (1977), 187-268.

[Bo] N. Bourbaki, Algèbre, Chap. 10: Algèbre homologique, Masson, Paris, 1980.

[Bur-O] D. Burghelea and C. Ogle, The Künneth formula in cyclic homology, Math. Z. 193 (1986), 527-536.

[GRW] S. Geller, L. Reid, and C. Weibel, The cyclic homology and K-theory of curves, J. Reine Angew. Math. (to appear).

[Go 1] T. Goodwillie, Relative algebraic K-theory and cyclic homology, Ann. of Math. (2) 124 (1986), 347-402.

[Go 2] __, On the general linear group and Hochschild homology, Ann. of Math. (2) 121 (1985), 383-407.

[Gr 1] D. Grayson, Products in K-theory and intersecting algebraic cycles, Invent. Math. 47 (1978), $71-83$.

[Gr 2] _-, Grothendieck rings and Witt vectors, Comm. Algebra 6 (1978), 249-255.

[GQ] _- Higher algebraic K-theory. II (after D. Quillen), Lecture Notes in Math., vol. 551, Springer-Verlag, Berlin, 1976.

[Gro] A. Grothendieck, Crystals and the de Rham cohomology of schemes, Dix Exposés sur la Cohomologie des Schémas, North-Holland, Amsterdam, 1968. 
[HJ] C. Hood and J. Jones, Some algebraic properties of cyclic homology groups, $K$-Theory 1 (1987), 361-384.

[Ig] K. Igusa, What happens to Hatcher and Wagoner's formula for $\pi_{0} C(M)$ when the first Postnikov invariant of $M$ is nontrivial?, Lecture Notes in Math., vol. 1046, Springer-Verlag, Berlin,

[III] L. Illusie, Complexe de de Rham-Witt et cohomologie cristalline, Ann. Sci. École Norm. Sup. (4) 12 (1979), 501-661.

[Ka] C. Kassel, Cyclic homology, comodules, and mixed complexes, J. Algebra 107 (1987), 195216.

[Laz] M. Lazard, Commutative formal groups, Lecture Notes in Math., vol. 443, Springer-Verlag, Berlin, 1975.

[LQ] J.-L. Loday and D. Quillen, Cyclic homology and the Lie algebra homology of matrices, Comment. Math. Helv. 59 (1984), 565-591.

[MS] H. Maazen and J. Steinstra, A presentation of $K_{2}$ of split radical pairs, J. Pure Appl. Algebra 10 (1977), 271-294.

[Q] D. Quillen, Higher algebraic $K$-theory. I, Lecture Notes in Math., vol. 341, Springer-Verlag, Berlin, 1973.

[St 1] J. Steinstra, Cartier-Dieudonne theory for Chow groups, J. Reine Angew. Math. 355 (1985), 1-66, and 362 (1985), 218-220.

[St 2] _ On $K_{2}$ and $K_{3}$ of truncated polynomial rings, Lecture Notes in Math., vol. 854, Springer-Verlag, Berlin, 1981.

[Wa] J. Wagoner, Delooping classifying spaces in algebraic K-theory, Topology 11 (1972), 349370.

[We 1] C. Weibel, Mayer-Vietoris sequences and module structures on $N K_{*}$, Lecture Notes in Math., vol. 966, Springer-Verlag, Berlin, 1982.

[We 2] _ _ K-theory and analytic isomorphisms, Invent. Math. 61 (1980), 177-197.

[We 3] _ Nil K-theory maps to cyclic homology, Trans. Amer. Math. Soc. 303 (1987), 541-558.

Department of Mathematics and Computer Science, Rutgers University, Newark, NEW JERSEY 07102 